\title{
Graded contrast echocardiography in pulmonary arteriovenous malformations
}

\author{
J.A. Parra*, J. Bueno", J. Zarauza", C. Fariñas-Alvarez ${ }^{+}$, J.M. Cuesta", \\ P. Ortiz ${ }^{\natural}$, R. Zarrabeitia ${ }^{\S}$, A. Pérez del Molino ${ }^{\S}$, M. Bustamante*, \\ L.M. Botella ${ }^{f}$ and M.T. Delgado**
}

ABSTRACT: To compare the results of transthoracic contrast echocardiography (TTCE) adding a grading scale with the results of thoracic computed tomography (CT) in order to optimise the use of both techniques.

95 patients with hereditary haemorrhagic telangiectasia (HHT) were examined with TTCE and thoracic CT to detect pulmonary arteriovenous malformations (PAVMs). According to previous studies, TTCE was divided into a four grade scale depending on the degree of opacification of the left ventricle after the administration of a contrast agent.

Of the 95 patients (50.5\% female; mean age 46 yrs), none with normal or grade 1 TTCE had detectable PAVMs on thoracic CT. Shunts of grades 2, 3 and 4 were associated with PAVMs according to thoracic CT in 25,80 , and $100 \%$ of the cases. There was a statistically significant association between the TTCE grade and the detection of a PAVM by thoracic CT. There were also statistically significant associations between TTCE grade and the cardiac cycle when the contrast was first visible in the left atrium, and size of the feeding artery.

Graded TTCE and timing of left atrium opacification may be useful techniques in selecting HHT patients for PAVM screening with thoracic CT scans.

KEYWORDS: Hereditary haemorrhagic telangiectasia, Osler-Weber-Rendu syndrome, pulmonary angiography, pulmonary arteriovenous malformations, thoracic computed tomography, transthoracic contrast echocardiography

ereditary haemorrhagic telangiectasia (HHT) or Osler-Weber-Rendu syndrome is a rare disorder (one in 5,000/8,000) $[1,2]$ transmitted in an autosomal dominant pattern and characterised by the progressive onset of epistaxis, mucocutaneous telangiectasias and vascular malformations that can develop in many organs, particularly the lung, where pulmonary arteriovenous malformations (PAVMs) are described in up to $48 \%$ of HHT patients [3]. According to the Curaçao criteria, the diagnosis of HHT is considered definite when three or more of the following criteria are present: spontaneous or recurrent epistaxis, multiple mucocutaneous telangiectasias, visceral vascular malformations or a first-degree relative with HHT [4]. The condition is suspected when only two of these features are present. Epistaxis is usually the earliest and most common manifestation of the disease.

Molecular genetic analysis has led to the identification of multiple HHT loci, with two genes (ENG and ACRVL1) being responsible for $90 \%$ of the described cases: ENG on the long arm of the chromosome 9 encoding for endoglin (type I HHT) and ACVRL1 on the long arm of the chromosome 12 coding for the activin receptor like kinase (ALK-1) (type II HHT) giving rise to haploinsufficiency, the proposed origin for the pathogenicity of the disease [5-7]. In addition, other genetic and environmental influences probably participate in the HHT phenotype [8]. Type I HHT has been associated with an increased risk of PAVMs while type II HHT with hepatic vascular malformations [8-10].

Patients with PAVMs are particularly at risk of severe local complications (spontaneous haemothorax or massive haemoptysis, especially in pregnancy) [11], dyspnoea and neurological complications, such as transient ischaemic attack (6-37\%), stroke (10-19\%) or cerebral abscess (5$19 \%$ ), due to the right to left shunt (RLS) that facilitates the passage of emboli into the cerebral circulation [12, 13]. Since these complications occur in asymptomatic individuals and can be effectively prevented by the safe procedure of embolotherapy, screening of asymptomatic HHT patients is recommended [14].

\section{AFFILIATIONS}

*Dept of Radiology, Hospital

Universitario Marqués de Valdecilla, University of Cantabria, IFIMAV,

**Dept of Radiology and Physical Medicine, University of Cantabria, Santander,

Depts of "Radiology,

"Cardiology,

+Preventive Medicine,

${ }^{\mathrm{s}}$ Internal Medicine, Hospital

Sierrallana, CIBERER, Torrelavega, and

${ }^{f}$ Centro de Investigaciones

Biológicas, CSIC (Council Research of Spain), CIBERER, Madrid, Spain.

CORRESPONDENCE

J.A. Parra

Servicio de Radiodiagnóstico

Hospital Universitario Marqués de Valdecilla

Avd. Valdecilla s/n. Santander 39008 Cantabria

Spain

E-mail: japarra@humv.es

Received:

July 042009

Accepted after revision:

Nov 032009

First published online:

Dec 082009

European Respiratory Journal Print ISSN 0903-1936 Online ISSN 1399-3003 
Several screening tests have been proposed alone or in combination (chest radiography, arterial oxygen measurements, radionuclide studies or transthoracic contrast echocardiography (TTCE)) $[3,15,16]$. The current recommendation is to use TTCE as the initial screening test for PAVMs [14]. TTCE has, however, two main drawbacks; the first is the high false positive rate when compared to computed tomography $(\mathrm{CT})$, probably in relationship with its ability to detect very small lesions [16, 17], and the second being from its incapacity to discriminate the size and localisation of the PAVMs, making it necessary to perform complementary tests.

For many years, pulmonary arteriography has been the gold standard for the study of patients under suspicion of having PAVMs. However, the development of multidetector technology, which allows the acquisition of multiple images in a short period of time, the possibility of image reconstruction in different planes and image analysis with different techniques (such us multiplanar reconstruction, maximum intensity projection or three-dimensional reconstructions), has converted CT to a powerful, sensitive and specific technique with a performance similar, or even superior, to angiography for the detection of pulmonary vascular disorders [18-21]. CT has, however, the drawback of using ionising radiation, which is recommended to be restricted, especially in young people.

Three recent articles [22-24] have emphasised the usefulness of a TTCE grading scale as a complementary tool to improve the selection of patients considered for a thoracic CT after TTCE in order to avoid unnecessary radiation exposure. Shunt grade on TTCE appears to be correlated with the presence of PAVMs on thoracic CT [24].

The purpose of this study was to compare the results of TTCE employing a graded scale with the results of thoracic CT in order to rationalise the utilisation of both techniques.

\section{MATERIALS AND METHODS}

A total of 125 HHT patients and their relatives were evaluated in the Sierrallana Hospital (Torrelavega, Spain) from June 2003 to June 2008. All patients were screened as part of the study protocol with a TTCE, thoracic CT and a genetic test. This study protocol was approved by the institutional review board.

\section{TTCE}

According to the established protocol, TTCE was performed by three experienced echocardiographers, by placing an intravenous line with a three-way stopcock to which two $10 \mathrm{~mL}$ syringes were connected. In the first studies, agitated saline solution was used $(9 \mathrm{~mL}$ of saline solution mixed with $0.5 \mathrm{~mL}$ of air and $0.5 \mathrm{~mL}$ of blood), and in the rest, $10 \mathrm{~mL}$ agitated fluid gelatine, without mixed air (Gelofusine $e$; Braun Medical AG, Emmenbrücke, Switzerland). With the patients in left lateral decubitus position, and using a four-chamber view, TTCE results were defined as positive for RLS if contrast solution was observed in the left atrium after injection without a Valsalva manoeuvre. The number of cardiac cycles before the appearance of bubbles in the left atrium, after their first appearance in the right atrium, was measured. The presence of bubbles after three cardiac cycles was considered a sign of PAVM as opposed to intracardiac shunt [15]. A second study with Valsalva manoeuvre was performed when contrast was present in the left atrium in less than four cardiac cycles. The study was considered positive for PAVM if intracardiac shunt were not visualised by colour Doppler, and the pattern of appearance of bubbles was not modified by Valsalva. For each type of study, the amount of contrast visible in the left ventricle was graded according to the grading system proposed by BARZILAI et al. [25] in four grades: grade 1 indicates minimal left ventricular opacity ( $<20$ bubbles), grade 2 indicates moderate opacity, grade 3 extensive opacity without outlining the endocardium and grade 4 extensive opacity with endocardial definition (fig. 1).

\section{Thoracic CT}

After performing an abdominal CT, a thoracic CT was made in a two detector CT with a section thickness of $3 \mathrm{~mm}$, and a reconstruction thickness of $1.5 \mathrm{~mm} ; 120 \mathrm{kV}$ and $100 \mathrm{mAs}$. A nonionic contrast agent $(300 \mathrm{mg}$ of iodine per $\mathrm{mL}$ with a rate of $3 \mathrm{~mL} \cdot \mathrm{s}^{-1}$ and a maximum dose of $2 \mathrm{~mL}$ per $\mathrm{kg}$ ) was administered routinely. In children, in whom abdominal CT was not performed, the study was made without the administration of a contrast agent with the $\mathrm{kV}$ and $\mathrm{mAs}$ adapted to the body habitus. Once the study was completed, it was evaluated by two experienced radiologists in interpreting thoracic CT for the presence of PAVMs, and the final diagnosis was reached by consensus. In all cases, the studies were evaluated in a work station in axial, multiplanar, maximum intensity projection and volume rendering reformations. The presence of a nodule with an afferent artery and efferent vein was considered diagnostic for lung PAVM on CT $[18,20]$. In all of these positive cases, the number of the PAVMs, the location in the lung (central or peripheral) [20] and the size of the feeding artery were recorded. Both TTCE and thoracic CT were performed and interpreted blinded to the results of the other study.

\section{Angiography}

After a preliminary study of the main, right and left pulmonary arteries, embolisation of the PAVM was performed, when possible, by positioning a microcatheter (Rebar-18 microcatheter, eV3; Microtherapeutics Inc., Irvine, CA, USA) in the feeding artery. Embolisation was performed with electrodetachable coils (GDC 360, 3D and Vortex microcoils; Boston Scientific Corporation, Natick, MA, USA).

\section{Statistical analysis}

The positive predictive value (PPV) of TTCE was calculated using thoracic CT as the reference standard. The PPV represents the percentage of the sample with a given TTCE grade that was determined to have PAVMs on CT. A Pearson Chi-squared test for association was performed for variables not following a normal distribution, and unpaired t-tests were used to evaluate the association between the amount of contrast visualized in the left ventricle, the cardiac cycle where the contrast was first visualised in the left atrium with TTCE, and the presence of PAVMs on CT.

Linear regression analysis was performed to determine if there was an association between the TTCE grade and the size of the feeding artery and efferent vein. All tests were two-tailed, and p-values $<0.05$ were considered significant. Statistical analyses were performed using SPSS v15.0 (SPSS Inc., Chicago, IL, USA). 

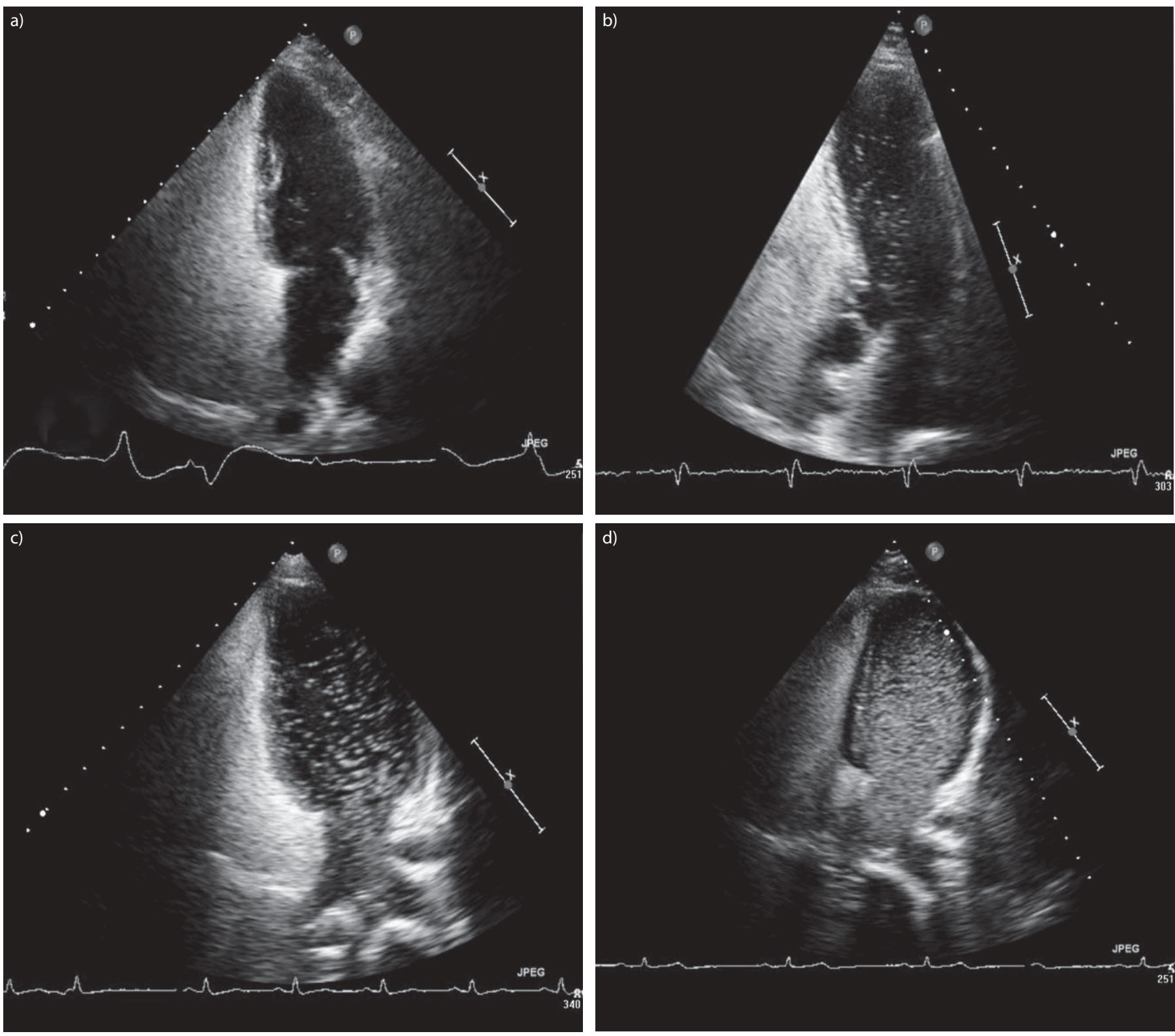

FIGURE 1. Transthoracic contrast echocardiography grades 1 to 4 with minimal (a), moderate (b), extensive (c) and extensive with endocardial definition (d) amount of contrast in left heart cavities.

\section{RESULTS}

Out of the 125 patients evaluated for suspected HHT, only those patients with three or four Curaçao criteria, and a TTCE, thoracic CT and angiography performed within an 180-day period were included in the study. Three patients with HHT (two type I HHT and one type II HHT) and with a TTCE diagnosis of patent foramen ovale (PFO) were also excluded. None of these patients with PFO have PAVMs on thoracic CT. 95 patients met all the criteria (table 1 ).

According to the thoracic CT findings 20 (21\%) of the 95 patients studied had PAVMs (fig. 2); 10 females, mean \pm SD age $41.1 \pm 14.4$ yrs (range 11-67 yrs). All 20 patients underwent a genetic study and $13(65 \%)$ were type I (table 2$)$.

In 15 patients we measured the size of the feeding artery in the thoracic CT (table 2). The mean \pm SD size of the feeding artery was of $3.78 \pm 1.08 \mathrm{~mm}$ (range $2.4-6.6 \mathrm{~mm}$ ). When we compared the size of the feeding artery with the TTCE grade, we found an association between both parameters that was statistically significant exclusively in the case of patients with a single PAVM. In these 12 patients, we found a significant relationship between the TTCE grade and the size of the feeding artery in a linear regression analysis, so that the bigger the size of the artery, the higher the TTCE grade, being significant according to the Pearson correlation $(0.6 ; \mathrm{p}=0.035)$ (table 3$)$.

TTCE was considered positive for extracardiac RLS in 71 $(74.7 \%)$ of the 95 patients. Of these 71 patients, 34 patients were considered grade 1, 20 grade 2, 10 grade 3 and seven grade 4 (table 4).

None of the patients with negative TTCE or grade 1 TTCE showed images compatible with PAVMs on thoracic CT. Five 


\begin{tabular}{|c|c|}
\hline TABLE 1 Characteristics of the study $p$ & \\
\hline Age yrs & $46.4(6-78)$ \\
\hline \multicolumn{2}{|l|}{ Sex } \\
\hline Female & 48/95 (50.5) \\
\hline Male & 47/95 (49.5) \\
\hline \multicolumn{2}{|l|}{ Curaçao criteria } \\
\hline Three & $54 / 95(56.8)$ \\
\hline Four & 41/95 (43.2) \\
\hline \multicolumn{2}{|l|}{ Genetic test $^{\#}$} \\
\hline Type I HHT & $33 / 90(36.7)$ \\
\hline Type II HHT & $55 / 90(61.1)$ \\
\hline No type I or type II HHT & $2 / 90(2.2)$ \\
\hline $\begin{array}{l}\text { Time between i.v. contrast injection and thoracic } \\
\text { CT image acquisition s }\end{array}$ & $103.0(80-144)$ \\
\hline Time between TTCE and thoracic CT days & $15.7(0-180)$ \\
\hline Time between thoracic CT and angiography days & $104.0(29-152)$ \\
\hline
\end{tabular}

( $25 \%$ ) of the 20 patients with grade 2 , eight $(80 \%)$ with grade 3 , and all $(100 \%)$ the patients with grade 4 showed PAVMs on thoracic CT. We found a significant association between TTCE grades and detection of PAVM on thoracic CT $(\mathrm{p}<0.0001)$. PPV was $0 \%(95 \%$ CI $0-10.2 \%)$ for grade $1 ; 25 \%$ (95\% CI $8.7-49.1 \%)$ for grade 2 ; $80 \%$ (95\% CI $44.7-97.5 \%$ ) for grade 3 ; and $100 \%$ (95\% CI $59-100 \%)$ for grade 4 . The sensitivity and negative predictive values of TTCE in our study were $100 \%$.

Conversely, we found a significant relationship $(\mathrm{p}<0.0001)$ between the cardiac cycle in which the contrast flow was first detected in the left atrium and TTCE grade, so that patients with higher TTCE grade showed an earlier left atrium opacification (table 5). In grades 2 and 3 the cardiac cycle was not useful to differentiate whether PAVMs were present or not on thoracic CT.

In the 10 patients with thoracic CT and angiography, we did not find differences in the number and location of the lesions between both techniques.

No significant adverse effects were observed in the patients related to the administration of the contrast agent, except for an 11-yr-old male with a PAVM who reported dizziness after the administration of the agitated fluid gelatine (grade 4 TTCE).

\section{DISCUSSION}

Early diagnosis of PAVMs in HHT patients is recommended, in order to prevent local or, more often, neurological complications [14]. Among the different screening techniques, TTCE (followed by CT in positive cases), is the most accepted screening method, given its low cost, accessibility, sensitivity and high negative predictive value of $90-100 \%[16,26]$. In according to these data, none of our patients with negative TTCE for extracardiac RLS showed PAVMs on thoracic CT.

However, TTCE shows problems derived from its high false positive rate when compared with $\mathrm{CT}$, probably related to its

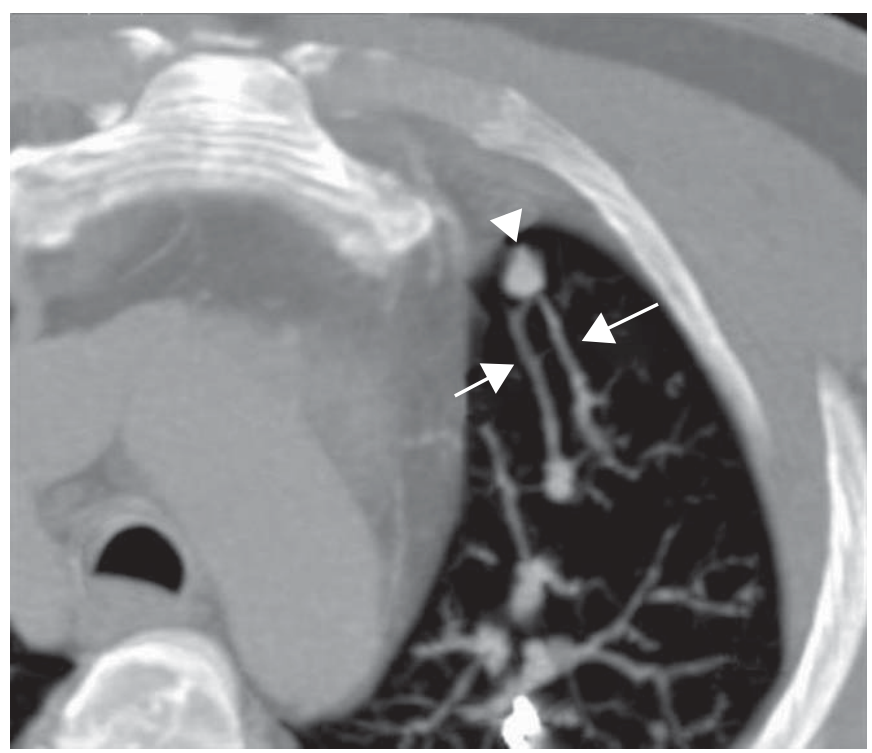

FIGURE 2. A 36-yr-old male with type I hereditary haemorrhagic telangiectasia and pulmonary arteriovenous malformations in the left upper lobe with a nodule (arrowhead), an afferent artery and efferent vein (arrows).

capability to detect unimportant PAVMs at a microscopic level $[16,17]$, a finding that may lead to further unnecessary CT investigation. In our study, only $20(28 \%)$ out of 71 patients with positive TTCE and a subsequent CT scan demonstrated PAVMs on thoracic CT. Similar results have been found by other investigators [22, 23]. Owing to these findings, several recent articles [22-24] have analysed the usefulness of adding a graded system to TTCE to improve the selection of patients for whom a further CT is necessary, in order to avoid unnecessary radiation exposure. Two grading systems have been used in these studies: one based on the classification model described by BARZILAI et al. [25] and followed by ZUKOTYNSKI et al. [22] and ourselves with a 4 grade scale, and another employed by GAZZANIGA et al. [23] and VAN GENT et al. [24] with a 3 grade scale, where grade 3 is equivalent to grades 3 and 4 in the scale of BARZILAI et al. [25]. Regardless of the grading scale used, all the studies have found a statistically significant correlation between the presence of detectable PAVMs on thoracic CT and TTCE grades, so that the probability of detecting PAVMs on thoracic CT is increased in higher TTCE grades.

If we compare our results in each TTCE grade with those obtained by other authors [22-24], we find that none of the patients with TTCE grade 1 in our series and in the GAZZANIGA et al. [23] series showed PAVMs on thoracic CT (using a similar cut-off to differentiate between TTCE grade 1 and 2). However, they were present in $2 \%$ of the patients in the study of ZUKOTYNSKI et al. [22] and in $22.9 \%$ in the VAN GENT et al. [24] study, the latter with a cut-off of 30 microbubbles. In TTCE grade 2, 3 and $4,25 \%, 80 \%$ and $100 \%$, respectively, of our patients had PAVMs. These results are in the range of these other studies, in which the incidence of PAVMs in grade 2 varies between $25 \%$ and $56 \%$ and in grade 3 or grade 3 and 4 , according to the classification scale used, between $56 \%$ and $100 \%$ [22-24]. 


\begin{tabular}{|c|c|c|c|c|c|c|c|c|c|}
\hline \multicolumn{2}{|c|}{ TABLE 2} & \multicolumn{8}{|c|}{$\begin{array}{l}\text { Characteristics of } 20 \text { patients with pulmonary arteriovenous malformations (PAVMs) on thoracic computed } \\
\text { tomography (CT) }\end{array}$} \\
\hline Patient & $\begin{array}{l}\text { Curaçao } \\
\text { criteria }\end{array}$ & $\begin{array}{c}\text { HHT genetic } \\
\text { type }\end{array}$ & $\begin{array}{l}\text { TTCE } \\
\text { grade }\end{array}$ & $\begin{array}{l}\text { Cardiac } \\
\text { cycle }^{\#}\end{array}$ & $\begin{array}{l}\text { PAVMs on } \\
\text { thoracic CT } n\end{array}$ & $\begin{array}{l}\text { PAVM: lung } \\
\text { location }\end{array}$ & $\begin{array}{l}\text { PAVM: lung lobe } \\
\text { location }\end{array}$ & $\begin{array}{l}\text { Afferent artery } \\
\text { size } \mathrm{mm}\end{array}$ & $\begin{array}{l}\text { Angiography/PAVM } \\
\text { lung lobe location }\end{array}$ \\
\hline 1 & 4 & $॥$ & 2 & 8 & 1 embolised & Peripheral & RLL & & \\
\hline 2 & 3 & । & 2 & 6 & 1 & Peripheral & RLL & 2.4 & \\
\hline 4 & 4 & । & 2 & 4 & 1 and 2 embolised & Peripheral & $\begin{array}{l}\text { Lingule (RLL; } \\
\text { LUL embolised) }\end{array}$ & 3.3 & \\
\hline 5 & 4 & $\|$ & 2 & 5 & 1 and 1 embolised & Peripheral & $\begin{array}{l}\text { Lingule (LLL } \\
\text { embolised) }\end{array}$ & 4.3 & \\
\hline 6 & 4 & $\|$ & 3 & 3 & 1 & Peripheral & RLL & 2.8 & \\
\hline 7 & 4 & $\|$ & 3 & 3 & 1 & Peripheral & LLL & 3 & \\
\hline 12 & 4 & $॥$ & 3 & 4 & 1 & Peripheral & RML & 4.3 & 1 PAVM/RML \\
\hline 13 & 4 & $\|$ & 3 & 4 & 1 & Peripheral & RLL & 5.3 & 1 PAVM/RLL \\
\hline 14 & 3 & $\|$ & 4 & 3 & 1 & Peripheral & RUL & 3.6 & \\
\hline 15 & 4 & । & 4 & & 1 and 1 embolised & Peripheral & RLL (LLL embolised) & 4 & 1 PAVM/RLL \\
\hline 16 & 4 & । & 4 & 3 & 1 & Peripheral & RLL & 6.6 & \\
\hline 17 & 4 & । & 4 & 4 & Multiple & & & & Multiple PAVMs \\
\hline 18 & 4 & 1 & 4 & 3 & $\begin{array}{c}\text { Multiple } \\
\text { (3 embolised) }\end{array}$ & & & & Multiple PAVMs \\
\hline 19 & 4 & । & 4 & 3 & $\begin{array}{l}\text { Multiple } \\
\text { (2 embolised) }\end{array}$ & & & & Multiple PAVMs \\
\hline 20 & 4 & I & 4 & 3 & Multiple & & & & \\
\hline
\end{tabular}

HHT: hereditary haemorrhagic telangiectasia; TTCE: transthoracic contrast echocardiography; RLL: right lower lobe; LUL: left upper lobe; LLL: left lower lobe; RUL: right upper lobe; RML: right median lobe; Embolised: PAVM previously treated with embolotherapy. ${ }^{*}$ : number of cardiac cycles when the contrast is first visualised in the left atrium.

These findings justify the need of a thoracic CT after positive TTCE in all patients with grade 3 or grade 3 and 4 where almost all of the patients will have a PAVM on CT and almost of them will be suitable for embolisation [24]. In patients with TTCE grade 2, only a reduced proportion will have a PAVM detected on CT and most of them will have a feeding artery too small to perform embolotherapy [24]. However, it still appears justified to perform a CT after TTCE because, as shown in our study, it is possible to find patients with a feeding artery of

\begin{tabular}{ll}
\hline TABLE 3 & $\begin{array}{l}\text { Relationships between transthoracic contrast } \\
\text { echocardiography (TTCE) grades and afferent } \\
\text { artery size on thoracic computed tomography in } \\
12 \text { patients with a single pulmonary } \\
\text { arteriovenous malformation }\end{array}$ \\
\hline TTCE grades & Afferent artery size mm \\
\hline Grade 2 TTCE & $2.7 \pm 0.4$ \\
Grade 3 TTCE & $3.7 \pm 0.8$ \\
Grade 4 TTCE & $5.1 \pm 2.1$ \\
\hline & \\
Data are presented as mean \pm SD.
\end{tabular}

$\geqslant 2 \mathrm{~mm}$. Although we did not find PAVMs on CT in patients with TTCE grade 1, it is feasible that they may be seen, as
TABLE 4 Relationship between graded transthoracic contrast echocardiography (TTCE) and thoracic computed tomography (CT) findings

Graded TTCE Patients n
on thoracic CT

\begin{tabular}{|c|c|c|}
\hline Negative TTCE & 24 & 0 \\
\hline Grade 1 & 34 & 0 \\
\hline \multirow[t]{2}{*}{ Grade 2} & 20 & $5(25 \%)$ \\
\hline & & 3 single ( 1 embolised) and 2 multiple \\
\hline \multirow[t]{2}{*}{ Grade 3} & 10 & $8(80 \%)$ \\
\hline & & 8 single \\
\hline \multirow[t]{2}{*}{ Grade 4} & 7 & $7(100 \%)$ \\
\hline & & 2 single and 5 multiple \\
\hline Total & 95 & 20 \\
\hline
\end{tabular}




\begin{tabular}{|c|c|c|c|}
\hline \multirow{2}{*}{$\begin{array}{l}\text { TABLE } 5 \\
\text { TTCE grade }\end{array}$} & \multicolumn{3}{|c|}{$\begin{array}{l}\text { Relationship between transthoracic contrast } \\
\text { echocardiography (TTCE) grades and the } \\
\text { cardiac cycle when the contrast was first } \\
\text { visualised in the left atrium }\end{array}$} \\
\hline & & Patients $\mathrm{n} / \mathrm{n}$ & $\begin{array}{c}\text { Cardiac cycle } \\
\text { (mean) }\end{array}$ \\
\hline \multicolumn{2}{|l|}{ Grade 1 TTCE } & $33 / 34$ & $7(6.9)$ \\
\hline \multicolumn{2}{|l|}{ Grade 2 TTCE } & $20 / 20$ & $5(5.2)$ \\
\hline \multicolumn{2}{|c|}{ Grade 3 TTCE } & $8 / 10$ & $4(3.6)$ \\
\hline \multicolumn{2}{|c|}{ Grade 4 TTCE } & $6 / 7$ & $3(3.2)$ \\
\hline \multicolumn{2}{|c|}{ Patients with PAVMs on thoracic CT } & $18 / 20$ & $4(3.94)$ \\
\hline \multicolumn{2}{|c|}{ Patients without PAVMs on thoracic CT } & $48 / 51$ & $6(6.26)$ \\
\hline
\end{tabular}

PAVM: pulmonary arteriovenous malformation; CT: computed tomography.

discussed previously [22, 24]. An important finding in our study, partially confirmed by the results of VAN GENT et al. [24] (where none of the patients with PAVMs on thoracic $\mathrm{CT}$ and a TTCE grade 1 were candidates for embolotherapy), is that there is an apparent relationship between the size of the feeding artery and TTCE grade. Although this was detected only in a small group of patients, we would expect that the few patients with grade 1 TTCE and a PAVM on thoracic CT will not have a feeding artery large enough to permit embolotherapy. Therefore, it could avoid in this group, the performance of a CT following TTCE or, the control studies with CT. However, more data are probably necessary before discontinuing thoracic CT after a positive TTCE for RLS.

According to the international guidelines for the diagnosis and management of HHT [14], all patients with a positive TTCE, including those with PAVMs not detectable on thoracic CT, should receive prophylactic antibiotics, especially before highrisk surgery and oral or dental manipulations [27].

In addition to the TTCE grade we investigated if the timing of contrast arrival in the left atrium could be helpful, to differentiate patients with or without detectable PAVMs on thoracic CT. In accordance with ZUKOTYNSKI et al. [22], we have not found a significant difference within TTCE grades 2 and 3 , to allow us to distinguish between patients with or without PAVMs on CT. Nevertheless, there was a statistically significant relationship between the cardiac cycle in which the contrast flow was localised in the left atrium and the TTCE grade, so that patients with higher grade had an earlier appearance of flow in the left atrium. These findings suggest that the moment of the visualisation of the contrast flow in the left atrium is dependent on the shunt intensity. The delay in the bubble detection in the left atrium after complete opacity of the right atrium is the parameter used to differentiate intracardiac from intrapulmonary shunts in TTCE. Traditionally, three or fewer cardiac cycles for intracardiac and four or more cardiac cycles for intrapulmonary RLS are used $[28,29]$. In our study, in agreement with ZUKOTYNSKI et al. [22], several patients with PAVMs on CT and TTCE grade 3 or 4 showed presence of flow in the left atrium in the third and fourth cardiac cycles. Although the presence of a PFO cannot be completely ruled out in these patients, as a transoesophageal echocardiogram was not carried out, the absence of flow through the interauricular septum by colour Doppler, and the absence of modification in the pattern of the appearance of bubbles with Valsalva, renders the existence of a right to left intracardiac shunt unlikely. The timing in which bubbles appear in the left atrium might be conditioned by the intensity of the shunt and sometimes could not be, as has been suggested, an accurate indicator of the localisation of RLS [22].

The safety of TTCE has been well documented elsewhere [30]. This procedure is generally well tolerated, with a low incidence of side-effects, all of them minimal and self-resolving [23]. We had only one patient with grade 4 TTCE and PAVM confirmed on thoracic CT that suffered a short-lived episode of dizziness.

Our results have some limitations, e.g. the small number of patients in whom PAVMs were detected and the fact that graded TTCE is a semi-quantitative technique. We considered, however, that a better definition of grade 1 and 2 should be achieved with a clear cut-off between these two grades. Differentiation between TTCE grades 3 and 4 appears less important because most of the patients will have a PAVM, and thoracic CT seems to be mandatory after TTCE. Another potential problem includes the inter-observer variability in the measurements of the feeding artery diameter when callipers are used on $\mathrm{CT}$, since it can over or underestimate the size of the measured vessel compared to angiography, although these differences do not appear significant [18].

\section{Conclusions}

Graded TTCE appears a useful technique for the reduction of CT studies, especially in grade 1 TTCE patients, although at present more studies are needed before generalising this indication. It also appears that, in patients with large PAVMs, there is a tendency for an earlier contrast flow appearance in the left atrium and an association between the size of the afferent artery and the TTCE grade.

\section{STATEMENT OF INTEREST}

None declared.

\section{ACKNOWLEDGEMENTS}

We thank C.L. Shovlin (NHLI Cardiovascular Sciences, Imperial College London, London, UK) for helpful comments and suggestions on the manuscript.

\section{REFERENCES}

1 Kjeldsen AD, Vase P, Green A. Hereditary haemorrhagic telangiectasia: a population-based study of prevalence and mortality in Danish patients. J Intern Med 1999; 245: 31-39.

2 Dakeishi M, Shioya T, Wada Y, et al. Genetic epidemiology of hereditary hemorrhagic telangiectasia in a local community in the northern part of Japan. Hum Mutat 2002; 19: 140-148.

3 Cottin V, Plauchu H, Bayle JY, et al. Pulmonary arteriovenous malformations in patients with hereditary hemorrhagic telangiectasia. Am J Respir Crit Care Med 2004; 169: 994-1000.

4 Shovlin CL, Guttmacher AE, Buscarini E, et al. Diagnostic criteria for hereditary hemorrhagic telangiectasia (Rendu-Osler-Weber syndrome). Am J Genet 2000; 91: 66-67.

5 Bossler AD, Richards J, George C, et al. Novel mutations in ENG and ACVRL1 identified in a series of 200 individuals undergoing clinical genetic testing for hereditary hemorrhagic telangiectasia 
(HHT): correlation of genotype with phenotype. Hum Mutat 2006; 27: 667-675.

6 Bourdeau A, Cymerman U, Paquet ME, et al. Endoglin expression is reduced in normal vessels but still detectable in arteriovenous malformations of patients with hereditary hemorrhagic telangiectasia type 1. Am J Pathol 2000; 156: 911-923.

7 Abdalla SA, Letarte M. Hereditary haemorrhagic telangiectasia: current views on genetics and mechanisms of disease. J Med Genet 2006; 43: 97-110.

8 Letteboer TG, Mager JJ, Snijder RJ, et al. Genotype-phenotype relationship in hereditary haemorrhagic telangiectasia. J Med Genet 2006; 43: 371-377.

9 Lesca G, Olivieri C, Burnichon N, et al. Genotype-phenotype correlations in hereditary hemorrhagic telangiectasia: data from the French-Italian HHT network. Genet Med 2007; 9: 14-22.

10 Kjeldsen AD, Møller TR, Brusgaard K, et al. Clinical symptoms according to genotype amongst patients with hereditary haemorrhagic telangiectasia. J Intern Med 2005; 258: 349-355.

11 Gershon AS, Faughnan ME, Chon KS, et al. Transcatheter embolotherapy of maternal pulmonary arteriovenous malformations during pregnancy. Chest 2001; 119: 470-477.

12 Cottin V, Chinet T, Lavole A, et al. Pulmonary arteriovenous malformations in hereditary hemorrhagic telangiectasia: a series of 126 patients. Medicine 2007; 86: 1-17.

13 Shovlin CL, Jackson JE, Bamford KB, et al. Primary determinants of ischaemic stroke/brain abscess risks are independent of severity of pulmonary arteriovenous malformations in hereditary haemorrhagic telangiectasia. Thorax 2008; 63: 259-266.

14 Faughnan ME, Palda VA, Garcia-Tsao G, et al. International Guidelines for the Diagnosis and Management of Hereditary Hemorrhagic Telangiectasia. J Med Genet 2009; [Epub ahead of print DOI: 10.1136/jmg.2009.069013].

15 Gossage J, Kanj G. Pulmonary arteriovenous malformations - a state of the art review. Am J Respir Crit Care Med 1998; 158: 643-661.

16 van Gent MW, Post MC, Luermans JG, et al. Screening for pulmonary arteriovenous malformations using transthoracic contrast echocardiography: a prospective study. Eur Respir J 2009; 33: 85-91.

17 Lee WL, Graham AF, Pugash RA, et al. Contrast echocardiography remains positive after treatment of pulmonary arteriovenous malformations. Chest 2003; 123: 351-358.

18 Remy J, Remy-Jardin M, Wattinne L, et al. Pulmonary arteriovenous malformations: evaluation with $\mathrm{CT}$ of the chest before and after treatment. Radiology 1992; 182: 809-816.
19 Duddalwar VA. Multislice CT angiography: a practical guide to $\mathrm{CT}$ angiography in vascular imaging and intervention. Br J Radiol 2004; 77: S27-S38.

20 Remy-Jardin M, Dumont P, Brillet PY, et al. Pulmonary arteriovenous malformations treated with embolotherapy: helical CT evaluation of long-term effectiveness after 2-21-year follow-up. Radiology 2006; 239: 576-585.

21 Nawaz A, Litt HI, Stavropoulos SW, et al. Digital subtraction pulmonary arteriography versus multidetector $\mathrm{CT}$ in the detection of pulmonary arteriovenous malformations. J Vasc Interv Radiol 2008; 19: 1582-1588.

22 Zukotynski K, Chan RP, Chow CM, et al. Contrast echocardiography grading predicts pulmonary arteriovenous malformations on CT. Chest 2007; 132: 18-23.

23 Gazzaniga P, Buscarini E, Leandro G, et al. Contrast echocardiography for pulmonary arteriovenous malformations screening: does any bubble matter? Eur J Echocardiogr 2009; 10: 513-518.

24 van Gent MW, Post MC, Snijder RJ, et al. Grading of pulmonary right-to-left shunt with transthoracic contrast echocardiography: does it predict the indication for embolotherapy? Chest 2009; 135: 1288-1292.

25 Barzilai B, Waggoner AD, Spessert C, et al. Two-dimensional contrast echocardiography in the detection and follow-up of congenital pulmonary arteriovenous malformations. Am J Cardiol 1991; 68: 1507-1510.

26 Morrell NW. Screening for pulmonary arteriovenous malformations. Am J Respir Crit Care Med 2004; 169: 978-979.

27 Shovlin C, Bamford K, Wray D. Post-NICE 2008: antibiotic prophylaxis prior to dental procedures for patients with pulmonary arteriovenous malformations (PAVMs) and hereditary haemorrhagic telangiectasia. Br Dent J 2008; 205: 531-533.

28 Frazin LJ. Patent foramen ovale or pulmonary arteriovenous malformation: an appeal for diagnostic accuracy. Chest 2007; 132: 5-6.

29 Soliman OI, Geleijnse ML, Meijboom FJ, et al. The use of contrast echocardiography for the detection of cardiac shunts. Eur J Echocardiogr 2007; 8: S2-S12.

30 Bommer WJ, Shah PM, Allen $\mathrm{H}$, et al. The safety of contrast echocardiography: report of the Committee on Contrast Echocardiography for the American Society of Echocardiography. J Am Coll Cardiol 1984; 3: 6-13. 